\title{
Recurrent pneumothoraces in a 65-year-old female: an unusual case of cystic lung disease
}

A 65-year-old female presented to the emergency department with a 3-day history of dyspnoea and rightsided chest discomfort. She had been an inpatient 6 weeks earlier with right-sided pneumothorax treated with a computed tomography-guided chest drain. On this admission her chest radiograph demonstrated a recurrence of the pneumothorax (fig. 1). She was a life long nonsmoker.

She had a pneumothorax on the left side 16 years earlier. Multiple pulmonary nodules were seen on imaging and she underwent thoracoscopy and a lung biopsy. A diagnosis of metastatic endometrial stromal sarcoma was made and she underwent a total abdominal hysterectomy and bilateral salpingo-oophorectomy. She then
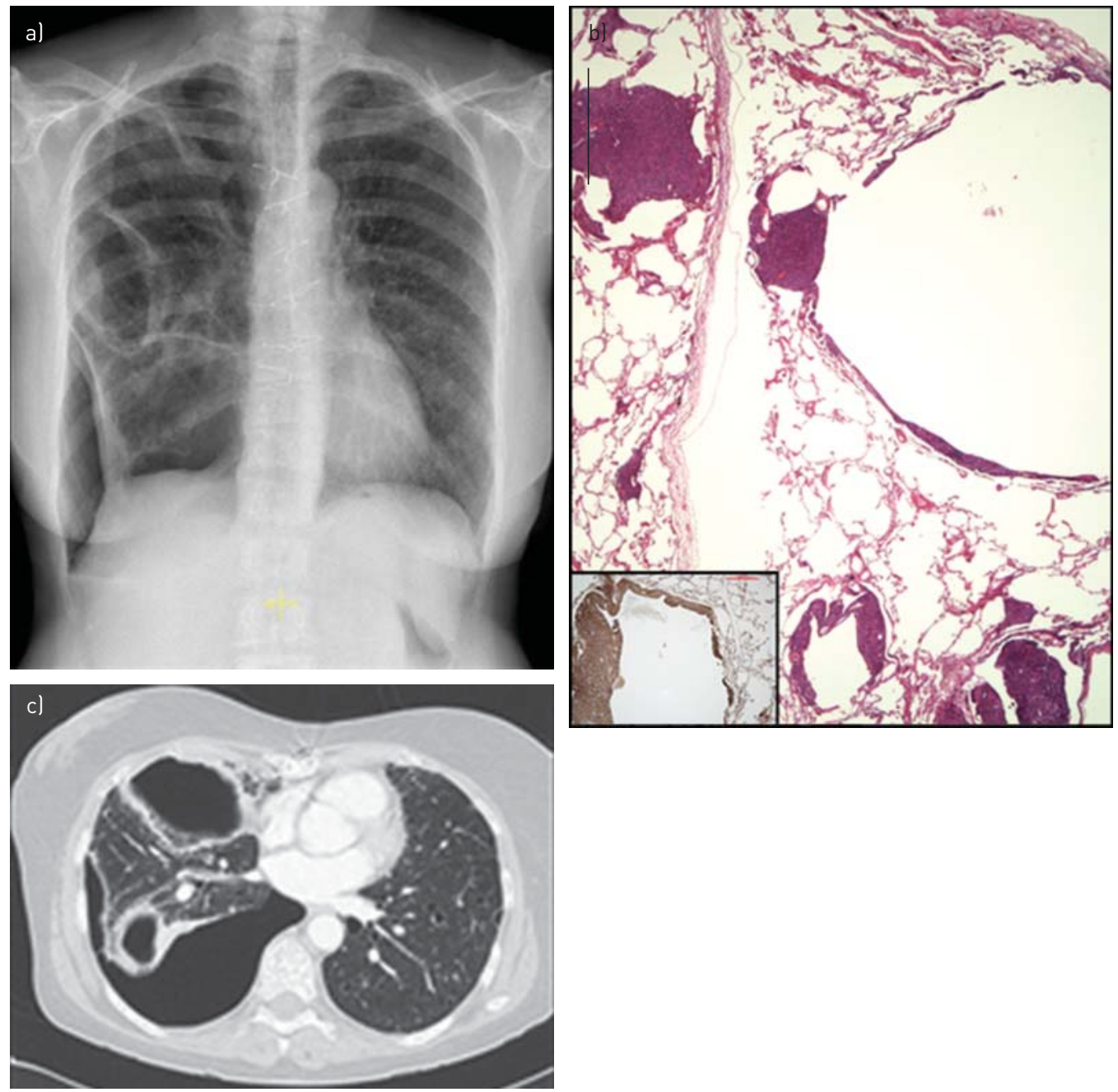

FIGURE 1 a) Chest radiograph showing a loculated right-sided pneumothorax. b) Histology of the lung tissue (from open lung biopsy) showed multiple small deposits, nodular or cystic, of spindle cells. Immunohistochemical markers were performed with negative staining for human melanoma black-45 monoclonal antibody and positive staining for progesterone receptor and CD10 (inset; scale bar $=500 \mu \mathrm{m}$ ). Scale bar $=1000 \mu \mathrm{m}$. c) Computed tomography of the thorax confirmed a large right-sided pneumothorax and a large cystic area in the right upper lobe. There were numerous smallwalled cysts in the left lung. 
began continued treatment with progesterone. 9 years ago she had bilateral pneumothoraces, which was treated by pleurectomy.

Uterine sarcomas are rare forms of uterine malignancy that arise from uterine smooth muscle and connective tissue. There are four main endometrial sarcomas: endometrial stromal sarcomas, leiomyosarcoma, rhabdomyosarcoma and undifferentiated endometrial sarcoma. Our patient had a low grade endometrial stromal sarcomas, which accounts for $\sim 15 \%$ of endometrial sarcomas. Preferred treatment is a total abdominal hysterectomy and bilateral salpingo-oophorectomy and hormonal therapy, with some centres advocating radiotherapy. Recurrences are quite common and patients can develop distant metastases after several years of tumour-free intervals [1]. The most commonly targeted site is the lung, with incidences between $7 \%$ and 28\% [2]. Pulmonary nodules or cysts can be multiple or solitary. Similarly lymphangioleiomyomatosis may present with recurrent pneumothoraces but can be differentiated on the basis of histopathological appearances, and staining for human melanoma black-45 monoclonal antibody in patients with lymphangioleiomyomatosis is usually positive [3].

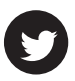

@ERSpublications

Cystic metastases should be considered in the differential of recurrent pneumothoraces http://ow.ly/udfrs

Sy Giin Chong, Patrick Mitchell, Aurelie Fabre and Timothy J. McDonnell

Dept of Respiratory Medicine and Pathology, St Vincent's University Hospital, Dublin, Ireland.

Correspondence: Timothy J. McDonnell, St Vincent's University Hospital, Elm Park, Dublin 4, Ireland.

E-mail: timothy.mcdonnell@ucd.ie

Received: July 152013 | Accepted after revision: July 192013

Conflict of interest: None declared.

Provenance: Submitted article, peer reviewed.

\section{References}

1 Aubry MC, Myers JL, Colby TV, et al. Endometrial stromal sarcoma metastatic to the lung: a detailed analysis of 16 patients. Am J Surg Pathol 2002; 26: 440-449.

2 Oliva E, Clement PB, Young RH. Endometrial stromal tumors: an update on a group of tumors with a protean phenotype. Adv Anat Pathol 2000; 7: 257-281.

3 Zhang X, Travis WD. Pulmonary lymphangioleiomyomatosis. Arch Pathol Lab Med 2010; 134: 1823-1828.

Eur Respir Rev 2014; 23: 271-272 | DOI: 10.1183/09059180.00005013 | Copyright (c)ERS 2014

ERR articles are open access and distributed under the terms of the Creative Commons Attribution Non-Commercial Licence 4.0. 